ESAIM: M2AN 47 (2013) 837-858

DOI: $10.1051 / \mathrm{m} 2 \mathrm{an} / 2012048$
ESAIM: Mathematical Modelling and Numerical Analysis

www.esaim-m2an.org

\title{
MACROSCOPIC CONTACT ANGLE AND LIQUID DROPS ON ROUGH SOLID SURFACES VIA HOMOGENIZATION AND NUMERICAL SIMULATIONS
}

\author{
S. Cacace $^{1}$, A. Chambolle ${ }^{2}$, A. DeSimone ${ }^{3}$ And L. Fedeli ${ }^{4}$
}

\begin{abstract}
We discuss a numerical formulation for the cell problem related to a homogenization approach for the study of wetting on micro rough surfaces. Regularity properties of the solution are described in details and it is shown that the problem is a convex one. Stability of the solution with respect to small changes of the cell bottom surface allows for an estimate of the numerical error, at least in two dimensions. Several benchmark experiments are presented and the reliability of the numerical solution is assessed, whenever possible, by comparison with analytical one. Realistic three dimensional simulations confirm several interesting features of the solution, improving the classical models of study of wetting on roughness.
\end{abstract}

Mathematics Subject Classification. 76D45, 74N30, 49S05, 49Q20, 65K15.

Received March 13, 2012. Revised August 7, 2012.

Published online March 10, 2013.

\section{INTRODUCTION}

Capillarity theory is a well established subject: the classical laws of Laplace and Young determining the shape of a drop in equilibrium on a solid surface have been obtained two centuries ago. Nevertheless, the study of sessile drops on rough surfaces has received considerable attention in the recent literature. This is mainly due to the fact that the intrinsic hydrophobic or hydrophilic properties of a given surface can be considerably enhanced by increasing its roughness (see Fig. 2). Some spectacular demonstrations of the effectiveness of roughness in producing super-hydrophobic or super-hydrophilic surfaces are shown in [15].

While describing metastable states and accounting for contact angle hysteresis in contact line dynamics is still quite challenging (see, however, $[2,6,10]$ for recent progress on these issues), the effect of roughness on the macroscopic contact angle exhibited by energy minimizing capillary drops is well understood, at least in principle.

Following the Gauss variational formulation of the problem of capillarity, the shape $L$ of a liquid drop of prescribed volume $\mathcal{V}$, sitting on a substrate $S$ and surrounded by vapor environment $V$, is obtained by minimizing

Keywords and phrases. Wetting, super-hydrophobic surfaces, contact-angle hysteresis, homogenization, total variation, nonsmooth optimization, augmented Lagrangian.

1 Sapienza Università di Roma, Piazzale Aldo Moro 5, 00185, Rome, Italy. cacace@mat.uniroma1.it

2 CMAP, Ecole Polytechnique, CNRS 91128, Palaiseau, France. antonin.chambolle@cmap.polytechnique.fr

3 SISSA-International School for Advanced Studies, Via Bonomea 265, 34136, Trieste, Italy. desimone@sissa.it

4 California Institute of Technology, 1200, E. California Blvd, 91125, Pasadena, CA. fedeli@caltech.edu 


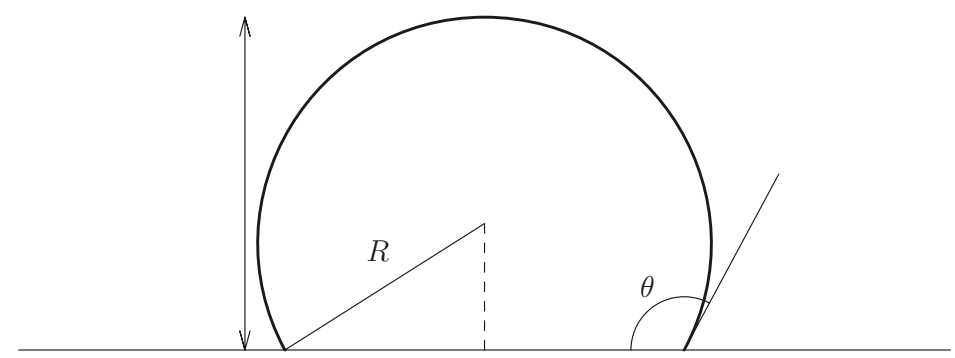

FiguRE 1. Sketch of a spherical cap.

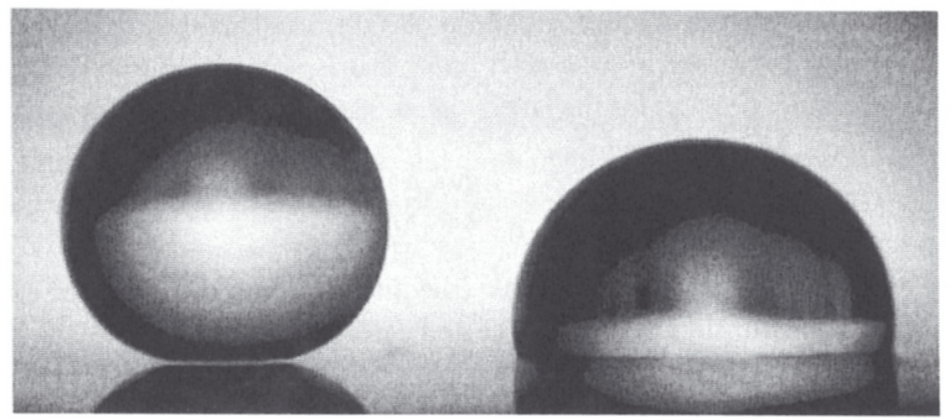

Figure 2. On the left a fakir drop in equilibrium on a bed of micro pillars (Cassie-Baxter configuration). If, after some evaporations, the radius of the drop is small enough, or external pressure is exerted on the globule, the drop switches to a Wenzel state (drop on the right [15]).

$$
F(L)=\sigma_{\mathrm{SL}}\left|\Sigma_{\mathrm{SL}}\right|+\sigma_{\mathrm{SV}}\left|\Sigma_{\mathrm{SV}}\right|+\sigma_{\mathrm{LV}}\left|\Sigma_{\mathrm{LV}}\right|,
$$

where $\Sigma_{X Y}$ is the interface between phases $X$ and $Y,\left|\Sigma_{X Y}\right|$ is its area and $\sigma_{X Y}$ is the corresponding interfacial energy density (surface tension).

Minimizers of (1.1) satisfy Laplace's law and Young's law on the contact angle (see Fig. 1), namely

$$
\cos \theta=\frac{\sigma_{\mathrm{SV}}-\sigma_{\mathrm{SL}}}{\sigma_{\mathrm{LV}}} .
$$

Let us define

$$
\begin{aligned}
& L_{\varepsilon}=\underset{|L|=\mathcal{V}}{\operatorname{argmin}} F_{\varepsilon}(L), \\
& F_{\varepsilon}=\sigma_{\mathrm{SL}}\left|\Sigma_{\mathrm{SL}}^{\varepsilon}\right|+\sigma_{\mathrm{SV}}\left|\Sigma_{\mathrm{SV}}^{\varepsilon}\right|+\sigma_{\mathrm{LV}}\left|\Sigma_{\mathrm{LV}}\right| .
\end{aligned}
$$

It has been shown [1] that, if the rough solid surface is an $\varepsilon$-periodic perturbation $S_{\varepsilon}$ of a flat surface $\mathrm{S}$, converging to $\mathrm{S}$ as $\varepsilon \rightarrow 0$ (i.e., in the limit in which the scale of roughness is vanishingly small compared to the true size of drop), then $F_{\varepsilon} \Gamma$-converges, as $\varepsilon \rightarrow 0$, to the homogenized energy

$$
F^{\text {hom }}=\sigma_{\mathrm{SL}}^{\text {hom }}\left|\Sigma_{\mathrm{SL}}\right|+\sigma_{\mathrm{SV}}^{\text {hom }}\left|\Sigma_{\mathrm{SV}}\right|+\sigma_{\mathrm{LV}}\left|\Sigma_{\mathrm{LV}}\right|,
$$

where $\sigma_{\mathrm{SL}}^{\text {hom }}$ and $\sigma_{\mathrm{SV}}^{\text {hom }}$ are obtained by suitable cell formulas and $L_{\varepsilon}$ converges towards $L$, given by

$$
L=\underset{|L|=\mathcal{V}}{\operatorname{argmin}} F^{\mathrm{hom}}(L) .
$$




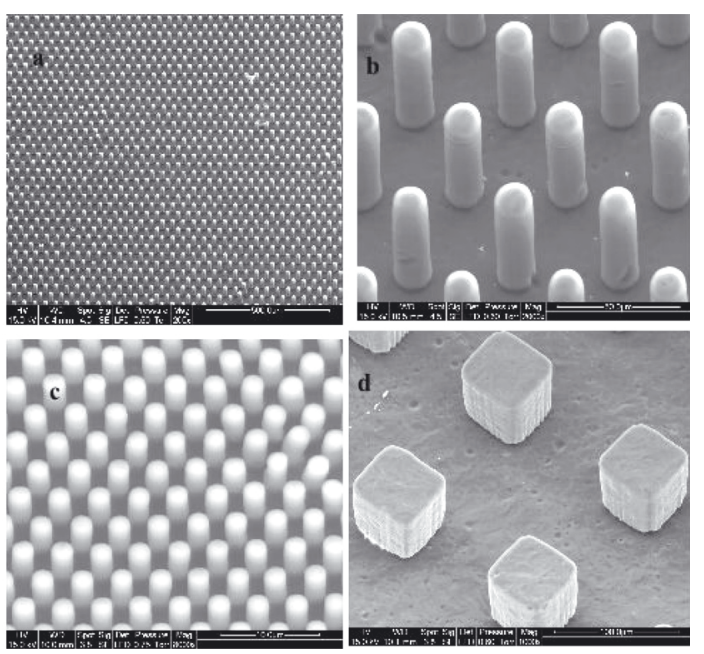

Figure 3. A thin glassy polymer film covered by a regular array of pillars, as seen by scanning electron microscopy (SEM) [17].

In particular, the macroscopic contact angle $\theta^{\text {hom }}$ satisfies

$$
\cos \theta^{\text {hom }}=\frac{\sigma_{\mathrm{SV}}^{\mathrm{hom}}-\sigma_{\mathrm{SL}}^{\mathrm{hom}}}{\sigma_{\mathrm{LV}}} .
$$

The renormalized surface tension $\sigma_{\mathrm{SV}}^{\text {hom }}$ (respectively $\sigma_{\mathrm{SL}}^{\text {hom }}$ ) represents the minimal energy per unit of macroscopic area for a transition layer between the microscopically rough solid and the vapor phase (respectively, the liquid phase). A physical explanation of the renormalization is that, for instance, the minimal energy transition between a sufficiently rough solid surface and the liquid phase can be realized by a composite interface in which the troughs near the bottom of the asperities are filled with vapor (see Fig. 2).

Moreover, the coefficients $\sigma_{\mathrm{SV}}^{\text {hom }}$ and $\sigma_{\mathrm{SL}}^{\text {hom }}$ can be characterized in principle as the solutions of well defined variational problems (see Sect. 2).

The goal of this paper is to propose a numerical algorithm for computing $\sigma_{\mathrm{SV}}^{\text {hom }}$ and $\sigma_{\mathrm{SL}}^{\text {hom }}$ (and hence $\theta^{\text {hom }}$ by (1.7)) in the case of a periodic rough surface of arbitrary complexity.

Complex micro-patterned surfaces, and even adaptive surfaces (see Fig. 3), are the subject of intense investigation in the recent chemical and physical literature. We believe that our approach will provide a very valuable predictive tool to guide the design of such surfaces.

The paper is organized as follows: in Section 2 we introduce the physical and mathematical setting of the cell problems in (1.5), giving results about existence and regularity of the solution. Section 3 contains numerical analysis on the approximation of the solution, in particular we provide an error estimate and a description of the discretization. We also introduce a suitable minimization algorithm and show how to adapt it to our framework. Finally, in Section 4, we perform some numerical experiments, both in two and three dimensions. In some particular cases of the two-dimensional setting, the analytical solution is available and we compare it with our numerical approximation. We complete the section with comments on the error obtained in the numerical tests and the one predicted in Section 3.

\section{AnAlysis of the CELL PROBlem}

This section is devoted to a physical and mathematical introduction to the problem. Here we mention the main physical aspects and we refer the interested reader to [1] for a detailed description. Then, we prove existence 
and regularity results for the solution of the problem. Finally, we provide an equivalent convex reformulation that, combined with stability results, opens the way to the numerical resolution.

\subsection{Homogenization formula and definition of the problem}

Let us focus on the hydrophobic case, i.e. $\cos \theta^{\text {hom }}<0$ (the hydrophilic case $\cos \theta^{\text {hom }}>0$ is similar and it is omitted in order to simplify the presentation). We assume that the solid surface is rough at a scale $\varepsilon>0$ and also flat at the macroscopic level, identified by the horizonal plane $\left\{x_{N}=0\right\}$ (for $N=2,3$ ).

To be precise, we define a microscopically rough solid $A_{\varepsilon}$ of the form

$$
A_{\varepsilon}:=\left\{\varepsilon x: x=\left(x_{1}, \ldots, x_{N}\right) \in A\right\},
$$

where $A$ is a closed set in $\mathbb{R}^{N}$ such that $\left\{x_{N} \leq 0\right\} \subset A \subset\left\{x_{N} \leq a\right\}$ for some $a>0$, it is $t$-periodic in the first $N-1$ directions for some $t>0$ (i.e. invariant under translation by $t e_{i}$ for $i=1, \ldots, N-1$ ) and it is symmetric with respect to the coordinate planes $\left\{x_{i}=0\right\}$ for $i=1, \ldots, N-1$.

In the limit $\varepsilon \rightarrow 0, A_{\varepsilon}$ converges to the half space $S:=\left\{x_{N} \leq 0\right\}$, whereas energy-minimizing configurations converge to the minimizers of the homogenized energy (1.5).

After a simple renormalization, we can reduce (1.1) to an equivalent interfacial energy of the form

$$
\widetilde{F}=|\cos \theta|\left|\Sigma_{\mathrm{SL}}\right|+\left|\Sigma_{\mathrm{LV}}\right| \text {. }
$$

The renormalized energy (2.2) shows that the only relevant physical parameter in the problem is $\cos \theta$. When $\cos \theta$ is negative, energy minimization promotes minimization of the area of the solid-liquid interface and $\partial S$ is called hydrophobic. In this situation (1.7) reduces to

$$
-\cos \theta^{\text {hom }}=\left|\cos \theta^{\text {hom }}\right|=\sigma_{\mathrm{SL}}^{\text {hom }}=\tilde{\sigma}_{\mathrm{SL}}=\inf _{V} \frac{\widetilde{F}\left(V, Q_{t}\right)}{\left|\omega_{t}\right|},
$$

where $\omega_{t}$ denotes the square in the plane $\left\{x_{N}=0\right\}$ such that $-t / 2<x_{i}<t / 2$ for $i=1, \ldots, N-1$ and $Q_{t}$ is the open cylinder $\omega_{t} \times \mathbb{R}$. Moreover, $\widetilde{F}\left(V, Q_{t}\right)$ denotes the energy associated with a test set $V$ within the periodicity cell $\omega_{t}$ and the infimum is taken over all bounded sets $V \subseteq Q_{t} \backslash A$ which are symmetric with respect to the coordinate planes $\left\{x_{i}=0\right\}$ for $i=1, \ldots, N-1$.

It is evident that the numerical study of the minimization problem in (2.3) yields the apparent macroscopic contact angle we are looking for.

From a mathematical point of view, we study this cell problem in $Q=\omega \times[0, H], H>0$, where $\omega=$ $\mathbb{R}^{N-1} / \mathbb{Z}^{N-1}$ is the $(N-1)$-dimensional torus (see Fig. 4). We choose a connected open set $A \subset Q$ (referred to as the "bottom surface") containing $\omega \times\{0\}$ and at a positive distance from $\Gamma=\omega \times\{H\}$ (in practice, a subgraph). To simplify, we assume that $A$ has Lipschitz boundary (sometimes this could be relaxed). The cell problem consists in finding a set $E \subset \Omega=Q \backslash \bar{A}$, containing $\Gamma$, that minimizes

$$
\bar{\sigma}=\min _{E} \operatorname{Per}(E, \Omega)+\int_{\partial A} \sigma \chi_{E} \mathrm{~d} \mathcal{H}^{N-1},
$$

where $\chi_{E}$ is the characteristic function of the set $E$ and $\operatorname{Per}(E, \Omega)=\int_{\Omega}\left|D \chi_{E}\right|$ is the perimeter of $E$ in $\Omega$, namely the Total Variation of $\chi_{E}$.

Here $\sigma$ is a constant such that $0<\sigma \leq 1$. More generally, we will also consider the case where $\sigma$ is a continuous function defined on $\partial A$, with values in $[0,1]$ and positive minimum.

In this setting, the set $E$ represents the volume occupied by the liquid and consists in the complement in $\Omega$ of the bounded set $V$ ("vapor") in (2.3). Here we assume that $H$ is large enough, so that $V \subset \Omega$.

The minimum value $\bar{\sigma}$ is just a simpler notation for the effective surface tension $\sigma_{\mathrm{SL}}^{\text {hom }}=\left|\cos \theta^{\text {hom }}\right|$ of the homogenized surface. 


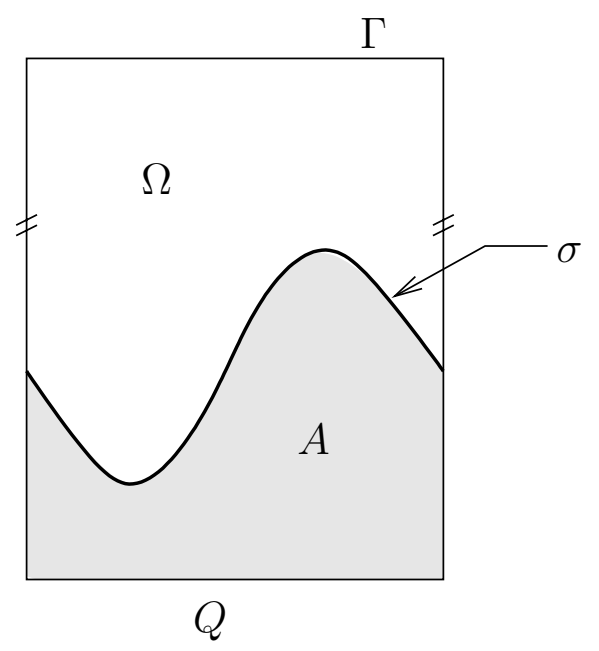

Figure 4. The setting of the cell problem.

Remark 2.1. In a problem such as (2.4), the Dirichlet boundary condition $\Gamma \subseteq E$ should be relaxed by adding a term $\int_{\Gamma}\left|1-\chi_{E}(x)\right| \mathrm{d} \mathcal{H}^{N-1}(x)$ in the functional, taking into account (in the perimeter term) the parts of $\Gamma$ where the trace of $\chi_{E}$ vanishes, which therefore must be considered as parts of the boundary of $E$. However observe that if $E$ is a minimizer of the relaxed problem and if $H^{\prime}<H$ is such that $\omega \times\left\{H^{\prime}\right\}$ is at positive distance from $\partial A$, then the set $E \cup\left(\omega \times\left(H^{\prime}, H\right]\right)$ has an energy lower than or equal to the relaxed energy of $E$, showing that in fact the initial (non relaxed) problem actually has a minimizer in the class of sets which contain $\Gamma$ (or, precisely, such that the trace of $\chi_{E}$ on $\Gamma$ is one).

\subsection{Existence of a solution}

The following lemma gives an existence result for our problem.

Lemma 2.2. Problem (2.4) has a solution.

The proof is given in [1] (Fig. 5 shows a possible solution). Here we provide a quick argument for the reader's convenience. The existence of a solution to (2.4) is an easy consequence of the lower semicontinuity (in $L^{1}$ ) of the functional which is minimized. This property can be shown as follows: let

$$
d_{A}(x)=\operatorname{dist}(x, A)-\operatorname{dist}(x, Q \backslash A)
$$

be the signed distance function to $\partial A$ and assume that $\sigma$ is extended to a continuous function $\sigma(x)$ in $Q$, such that $0<\sigma<1$ in $Q \backslash \partial A$. We define

$$
\psi(t)= \begin{cases}t^{2} /\left(1+t^{2}\right) & \text { if } \quad t \geq 0 \\ t^{2} & \text { otherwise. }\end{cases}
$$

The functional in (2.4) can be rewritten as the supremum

$\sup _{n \geq 1} \int_{Q}\left(\sigma(x)+\psi\left(n d_{A}(x)\right)(1-\sigma(x))\right)\left|D \chi_{E}\right|+\int_{\Gamma}\left|1-\chi_{E}(x)\right| \mathrm{d} \mathcal{H}^{N-1}(x)+\int_{\omega \times\{0\}} \psi\left(n \mathrm{~d}_{A}(x)\right) \chi_{E}(x) \mathrm{d} \mathcal{H}^{N-1}(x)$

if $E$ is a finite perimeter set in $Q$, whereas it is $+\infty$ if $E \cap A$ has positive measure. Then, it is easy to see that, for every $n$, each functional in this supremum is lower semicontinuous and this implies the thesis. 


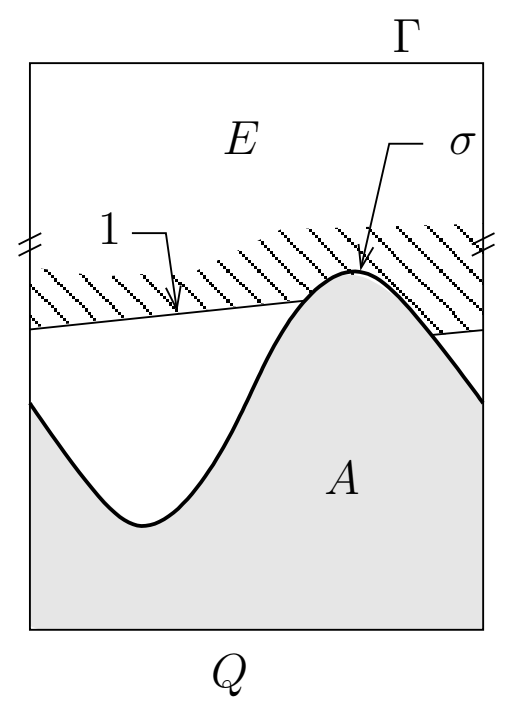

Figure 5. A possible solution $E$.

\subsection{Basic regularity properties}

We recall that, by assumption, the boundary $\partial A$ is at least of Lipschitz regularity. Moreover, we remark that the boundary $\partial E$ of any minimizer $E$ is a minimal surface in $\Omega$, hence it is analytical (if the dimension is not too high, as in the cases $N=2,3$ we are interested in). In particular, $\overline{\partial E \cap \Omega} \subset \partial E \cup \partial A$ and then $\partial E \cup \partial A$ is a closed set of finite measure $\mathcal{H}^{N-1}$ (covered by finitely many Lipschitz graphs). It follows that

$$
\frac{|\{\operatorname{dist}(\cdot, \partial E \cup \partial A) \leq \delta\}|}{2 \delta} \stackrel{\delta \rightarrow 0}{\longrightarrow} \mathcal{H}^{N-1}(\partial E \cup \partial A)
$$

(see $[3,11]$ for the convergence of Minkowski contents).

The study of the contact surface $\partial\left\{x \in \partial A: \chi_{E}(x)=1\right\}$ would be interesting. In particular, it is likely that if $\partial A$ is smooth enough, then the contact surface will have some regularity $[4,12,16]$. For a piecewise affine $\partial A$ this is less clear and we leave this point for future study. This will be important to get a complete proof of the error estimate in dimension higher than $N=2$ (see Sect. 3).

We observe, however, that a straightforward and standard estimate (see for instance [5]) bounds from below the density of $E$ at points in $\bar{E}$ (the closure being understood here in $Q$ ). To get rid of any ambiguity, we identify $E$ with the set $E^{1}$ of the points where its Lebesgue density is 1 . The density estimates will show that this is an open set in $Q$, as well as the set of points $E^{0}$ of density 0 . The complement $Q \backslash\left(E^{1} \cup E^{0}\right)$, which is known as the set-theoretical boundary (and is Lebesgue-negligible), therefore coincides with its topological boundary (and $\mathcal{H}^{N-1}$-a.e. with its reduced boundary, see [11]).

The estimate is obtained as follows. We assume, here and in the remainder of the paper, that $\sigma>0$ is either a constant or a function bounded from below by a constant $\sigma_{0}>0$. Consider a point $x \in \bar{E}$ such that $|B(x, r) \cap E|>0$ for each $r>0$ (otherwise $x$ is in the interior of the points of density 0 for $E$ ). Here $B(x, r$ ) denotes the ball of center $x$ and radius $r$, and we assume that $r$ is small enough so that $B=B(x, r) \subset Q$. More precisely, we should consider a ball in the periodic "unfolding" of $Q$ in $\mathbb{R}^{N-1} \times[0, H]$, whose canonical projection onto $\omega \times[0, H]$ is $Q$, and consider only balls $B$ which lie inside one period (hence, $r \leq 1$ ). But, since we think there is no ambiguity, we will skip this detail to make the proof more readable. 
Then, for a.e. $r \in(0,1)$,

$$
\operatorname{Per}(E \cap B)=\mathcal{H}^{N-1}(\partial B \cap E)+\mathcal{H}^{N-1}(\partial E \cap B \cap \Omega)+\int_{\partial A \cap B} \chi_{E} \mathrm{~d} \mathcal{H}^{N-1}
$$

and, by the minimality of $E$, i.e.

$$
\operatorname{Per}(E, \Omega)+\sigma_{0} \int_{\partial A} \chi_{E} \mathrm{~d} \mathcal{H}^{N-1} \leq \operatorname{Per}(E \backslash B, \Omega)+\sigma_{0} \int_{\partial A} \chi_{E \backslash B} \mathrm{~d} \mathcal{H}^{N-1},
$$

it follows that

$$
\mathcal{H}^{N-1}(\partial B \cap E) \geq \mathcal{H}^{N-1}(\partial E \cap B \cap \Omega)+\sigma_{0} \int_{\partial A \cap B} \chi_{E} \mathrm{~d} \mathcal{H}^{N-1} .
$$

Combining the last two inequalities and using the isoperimetric inequality, we get

$$
c|E \cap B|^{1-\frac{1}{N}} \leq \frac{\sigma_{0}+1}{\sigma_{0}} \mathcal{H}^{N-1}(\partial B \cap E) .
$$

Letting $f(r):=|E \cap B(x, r)|$, so that $f^{\prime}(r)=\mathcal{H}^{N-1}(\partial B \cap E)$ for a.e. $r$, we deduce (using Gronwall's lemma) that there exists a constant $\kappa>0$, depending only on the dimension and $\sigma_{0}$, such that $f(r) / r^{N} \geq \kappa>0$. In particular, if $|E \cap B(x, r)|<\kappa r^{N}$ for some small radius $(\leq 1)$, then $x$ is in the interior of the complement of $E$. It follows that the set $E^{0}$ of points of $Q$ where $E$ has density zero is open.

The same kind of argument would show that if $x \in \Omega \backslash E$ and $B(x, r) \subset \Omega$, then $|B(x, r) \backslash E| \geq \kappa r^{N}$. Since we have assumed that $\partial A$ is Lipschitz, we can easily prove (possibly changing the value of $\kappa$ ) that if $x \in Q \backslash E$ and $|B(x, r) \backslash E|<\kappa r^{N}$, then $x$ is in the interior of $E^{1}$. We deduce that the topological boundary of $E$ consists exactly of the points of $Q$ where $E$ has density neither 0 nor 1 . We have shown the following:

Lemma 2.3. Let $E$ solve (2.4). Then $E$, as a subset of $Q$, satisfies the two following density estimates: there exists $\kappa>0$ such that, for $r$ small enough $(r \leq 1$ and such that $B(x, r) \subset Q)$,

- if $|E \cap B(x, r)| \leq \kappa r^{N}$, then there is a smaller radius $r^{\prime}>0$ such that $\left|E \cap B\left(x, r^{\prime}\right)\right|=0$,

- if $|B(x, r) \backslash E| \leq \kappa r^{N}$, then there is a smaller radius $r^{\prime}>0$ such that $\left|B\left(x, r^{\prime}\right) \backslash E\right|=0$.

In particular, the points of Lebesgue density 0 or 1 form two open sets, with common topological boundary (denoted by $\partial E$ ), which coincides $\mathcal{H}^{N-1}$-a.e. with the reduced boundary of $E$ in $Q$.

\subsection{Equivalent convex formulation}

Here we show that our minimization problem is a convex problem, i.e. a minimization of a convex functional over a convex domain. The convex formulation will be useful to perform the numerical discretization of the problem and implement the algorithm proposed for its resolution (see Sect. 3).

The approach is standard. Let $u \in B V(\Omega)$ and consider the problems

$$
\bar{\sigma}_{1}=\min \left\{\int_{\Omega}|D u|+\int_{\partial A} \sigma u \mathrm{~d} \mathcal{H}^{N-1}: u \in B V(\Omega), u=1 \quad \text { on } \quad \Gamma, u \geq 0\right\}
$$

and

$$
\bar{\sigma}_{2}=\min \left\{\int_{\Omega}|D u|+\int_{\partial A} \sigma|u| \mathrm{d} \mathcal{H}^{N-1}: u \in B V(\Omega), u=1 \quad \text { on } \quad \Gamma\right\}
$$

The following proposition shows that $\bar{\sigma}_{1}=\bar{\sigma}_{2}=\bar{\sigma}$, where $\bar{\sigma}$ is given by (2.4).

Proposition 2.4. We have $\bar{\sigma}_{1}=\bar{\sigma}_{2}=\bar{\sigma}$. Moreover, given any solution $E$ of (2.4), then $\chi_{E}$ solves both (2.6) and (2.7). Conversely, given any solution $u$ of either (2.6) or (2.7), then for any $s \in(0,1),\{u>s\}$ and $\{u \geq s\}$ are both solutions of (2.4). 
Remark 2.5. As discussed in Remark 2.1, we should relax the boundary condition $u=1$ on $\Gamma$, by adding a term $\int_{\Gamma}|1-u(x)| \mathrm{d} \mathcal{H}^{N-1}(x)$ to the functionals (2.6) and (2.7). Again, for any function $u$, we can always define

$$
u^{\prime}(x)= \begin{cases}u(x) & \text { if } \quad x_{N}<H^{\prime} \\ 1 & \text { otherwise }\end{cases}
$$

which has an energy less than or equal to the energy of $u$, showing that the non-relaxed problem can indeed be minimized in the class of functions which are 1 in a neighborhood of $\Gamma$, where it is equivalent to the relaxed problem.

Proof of Proposition 2.4. The proof is an easy consequence of the coarea formula. First, the value $\bar{\sigma}$ of (2.4) is greater than or equal to the solutions of the two other minimization problems. Indeed, if $E$ is a set with finite perimeter in $\Omega$, then the energy of $E$ is the same as the energy of $\chi_{E}$ in (2.6) and (2.7). It follows that if $u$ is a solution of (2.7), then $u \geq 0$ a.e. (and also $u \leq 1$, since otherwise $(0 \vee u) \wedge 1$ has lower energy, strictly lower if it differs from $u$ ), so that it is a solution of (2.6). Now, we also have

$$
\int_{\Omega}|D u|+\int_{\partial A} \sigma u \mathrm{~d} \mathcal{H}^{N-1}=\int_{0}^{1}\left(\operatorname{Per}(\{u>s\}, \Omega)+\int_{\partial A} \sigma \chi_{\{u>s\}} \mathrm{d} \mathcal{H}^{N-1}\right) \mathrm{d} s \geq \bar{\sigma}
$$

and this shows that the value $\bar{\sigma}_{1}=\bar{\sigma}_{2}$ is greater or equal to the value of (2.4). In particular $\{u>s\}$ solves (2.4) for a.e. $s \in(0,1)$. But, since $\{u>s\}=\bigcup_{n}\left\{u>s_{n}\right\}$ for any sequence $s_{n} \downarrow s$ and $\{u \geq s\}=\bigcap_{n}\left\{u>s_{n}\right\}$ for any sequence $s_{n} \uparrow s$, the conclusion follows by approximation.

\subsection{Comparison}

We show that our problem is monotone with respect to $\sigma$. In particular, the solution is generically unique, in the sense that if we replace $\sigma$ in (2.4) by $\sigma+t, t \in \mathbb{R}$, then there is a unique minimizer $E_{t}$ for all $t$ but a countable number.

Lemma 2.6. Let $\sigma<\sigma^{\prime}$ on $\partial A$. Assume that $E$ solves (2.4) with $\sigma$ and $E^{\prime}$ solves the same problem with $\sigma$ replaced by $\sigma^{\prime}$. Then $\partial E \cap \partial A \supseteq \partial E^{\prime} \cap \partial A$.

In particular, if $E$ is the largest (in the sense of the inclusion) solution corresponding to $\sigma$ and $E^{\prime}$ is the smallest solution corresponding to $\sigma^{\prime}$, then $E \supseteq E^{\prime}$.

Proof. Let $u$ and $u^{\prime}$ respectively solve (2.6) with $\sigma$ and $\sigma^{\prime}$. In particular,

$$
\begin{aligned}
& \int_{\Omega}|D u|+\int_{\partial A} \sigma u \mathrm{~d} \mathcal{H}^{N-1} \leq \int_{\Omega}\left|D\left(u \vee u^{\prime}\right)\right|+\int_{\partial A} \sigma\left(u \vee u^{\prime}\right) \mathrm{d} \mathcal{H}^{N-1}, \\
& \int_{\Omega}\left|D u^{\prime}\right|+\int_{\partial A} \sigma^{\prime} u^{\prime} \mathrm{d} \mathcal{H}^{N-1} \leq \int_{\Omega}\left|D\left(u \wedge u^{\prime}\right)\right|+\int_{\partial A} \sigma^{\prime}\left(u \wedge u^{\prime}\right) \mathrm{d} \mathcal{H}^{N-1} .
\end{aligned}
$$

Summing up both inequalities and using the celebrated inequality

$$
\int_{\Omega}\left|D\left(u \vee u^{\prime}\right)\right|+\left|D\left(u \wedge u^{\prime}\right)\right| \leq \int_{\Omega}|D u|+\left|D u^{\prime}\right|,
$$

we get

$$
\int_{\partial A} \sigma^{\prime}\left(u^{\prime}-u \wedge u^{\prime}\right) \mathrm{d} \mathcal{H}^{N-1} \leq \int_{\partial A} \sigma\left(u \vee u^{\prime}-u\right) \mathrm{d} \mathcal{H}^{N-1}
$$

Since $u^{\prime}-u \wedge u^{\prime}=u \vee u^{\prime}-u=\left(u^{\prime}-u\right)^{+}$, we deduce that if $\sigma<\sigma^{\prime}$, then $\left(u^{\prime}-u\right)^{+}=0 \mathcal{H}^{N-1}$-a.e. on $\partial A$. In other words, the traces of $u$ and $u^{\prime}$ on $\partial A$ satisfy $u^{\prime} \leq u$. It follows that $\partial E^{\prime} \cap \partial A \subseteq \partial E \cap \partial A$.

If $u^{\prime}$ is a minimal solution and $u$ is a maximal solution, we also deduce that $u^{\prime} \leq u$ a.e. in $\Omega$, since otherwise $u^{\prime} \wedge u \leq u^{\prime}$ would have energy less than or equal to the energy of $u^{\prime}$ in (2.6), and the same for $u^{\prime} \vee u \geq u$ with respect to $u$. 
Remark 2.7. In dimension $N=2$, if $\partial A$ is a graph and $u=\chi_{E}$ is a solution of the problem, then it follows that $\partial E$ is also a graph. In particular, for a given trace $u=\chi_{\partial E \cap \partial A}$ on $\partial A$, the graph $\partial E \cap \Omega$ is unique, as it is the solution of a strictly convex problem. In this case, $\sigma<\sigma^{\prime} \Rightarrow E \supseteq E^{\prime}$.

\subsection{Stability of the cell problem}

Here we show that if $\partial A$ is Lipschitz, then the cell problem is "continuous" with respect to variations of $\partial A$, provided that the measure of $\partial A$ is continuously changed.

Proposition 2.8. For $n \rightarrow \infty$, let $A_{n} \rightarrow A$ be such that $\partial A_{n} \rightarrow \partial A$ in the Hausdorff sense and $\mathcal{H}^{N-1}\left(\partial A_{n}\right) \rightarrow$ $\mathcal{H}^{N-1}(\partial A)$. Assume that $\sigma_{n}: Q \rightarrow[0,1]$ is a continuous function, converging uniformly to $\sigma$. Assume also that the boundary $\partial A$ is Lipschitz and define

$$
\mathcal{E}(u)=\int_{\Omega}|D u|+\int_{\partial A} \sigma|u| \mathrm{d} \mathcal{H}^{N-1} .
$$

Moreover, set $\Omega_{n}=Q \backslash \bar{A}_{n}$ and define

$$
\begin{gathered}
\mathcal{E}_{n}(u)=\int_{\Omega_{n}}|D u|+\int_{\partial A_{n}} \sigma_{n}|u| \mathrm{d} \mathcal{H}^{N-1}, \\
\bar{\sigma}_{n}=\min _{E \supset \Gamma} \mathcal{E}_{n}\left(\chi_{E}\right),
\end{gathered}
$$

where $\bar{\sigma}_{n}$ is the effective surface tension associated to $A_{n}$ and $\sigma_{n}$.

Then, for $n \rightarrow \infty$,

$$
\bar{\sigma}_{n} \rightarrow \bar{\sigma}=\min _{E \supset \Gamma} \mathcal{E}\left(\chi_{E}\right) .
$$

Remark 2.9. The assumption that $\partial A$ is Lipschitz could be replaced by a slightly weaker assumption, such as the fact of being locally a subgraph at each point.

Proof of Proposition 2.8. We show a $\Gamma$-convergence result: first we extend $\mathcal{E}_{n}$ and $\mathcal{E}$ to $B V(Q)$, by defining (with a little abuse of notation)

$$
\mathcal{E}_{n}(u)=\left\{\begin{array}{lll}
\mathcal{E}_{n}\left(u_{\mid \Omega_{n}}\right) & \text { if } \quad u=0 & \text { o.e. in } \quad A_{n} \\
+\infty & \text { otherwise }
\end{array}\right.
$$

and

$$
\mathcal{E}(u)=\left\{\begin{array}{ll}
\mathcal{E}\left(u_{\mid \Omega}\right) & \text { if } \quad u=0 \\
+\infty & \text { otherwise }
\end{array} \text { a.e. in } A .\right.
$$

Let $u_{n} \rightarrow u$. For every $B \subset \subset \Omega$, we have that $B \subset \Omega_{n}$ for $n$ large enough and

$$
\int_{B}|D u| \leq \liminf _{n \rightarrow \infty} \int_{B}\left|D u_{n}\right| .
$$

If $B$ is a neighborhood of $\partial A$, then it is also a neighborhood of $\partial A_{n}$ for $n$ large enough and

$$
\begin{aligned}
\int_{\partial A} \sigma|u| \leq \int_{B} \sigma|D u| & \leq \liminf _{n \rightarrow \infty} \int_{B} \sigma_{n}\left|D u_{n}\right| \\
& \leq \liminf _{n \rightarrow \infty} \int_{\partial A} \sigma_{n}\left|u_{n}\right|+\int_{B \cap \Omega_{n}}\left|D u_{n}\right| .
\end{aligned}
$$

From (2.8) and (2.9) we easily deduce the $\Gamma$ - lim inf inequality, namely

$$
\mathcal{E}(u) \leq \liminf _{n \rightarrow \infty} \mathcal{E}_{n}\left(u_{n}\right) .
$$


Conversely, let $u \in B V(\Omega ;[0,1])$ (identified by $u \chi_{\Omega} \in B V(Q ;[0,1])$ ), such that $u=1$ on $\Gamma$. We want to find a sequence $u_{n}$ (also satisfying $u_{n}=1$ on $\Gamma$ ), converging to $u$ and such that the $\Gamma$-lim sup inequality holds, i.e.,

$$
\limsup _{n \rightarrow \infty} \mathcal{E}_{n}\left(u_{n}\right) \leq \mathcal{E}(u)
$$

From both (2.10) and (2.11) it will follow the $\Gamma$-convergence of $\mathcal{E}_{n}$ to $\mathcal{E}$, yielding $\bar{\sigma}_{n} \rightarrow \bar{\sigma}$.

Now, by Meyers-Serrin's theorem, there exists $u_{k} \rightarrow u$ such that $u_{k} \in C^{\infty}(\Omega ;[0,1])$ and

$$
\int_{\Omega}\left|\nabla u_{k}\right| \mathrm{d} x \rightarrow \int_{\Omega}|D u| \quad \text { as } k \rightarrow \infty .
$$

Since, by construction, the traces of $u_{k}$ and $u$ coincide on $\partial A$ (and in any case, since $\partial A$ is Lipschitz, the trace of $u_{k}$ goes to the trace of $u$ as a consequence of the convergence of the total variations), then $\mathcal{E}\left(u_{k}\right) \rightarrow \mathcal{E}(u)$ as $k \rightarrow \infty$ and (by a standard diagonal argument) it is enough to show that (2.11) holds for each $u_{k}$ : hence we assume that $u$ is smooth in $\Omega$.

Since $\partial A$ is Lipschitz, we can extend $u_{\mid \Omega}$ to a function $u^{\prime}$ defined on a slightly larger set $\Omega^{\prime}=\{x \in Q$ : $\operatorname{dist}(x, \Omega)<\delta\}$, for $\delta>0$, in such a way that $0 \leq u^{\prime} \leq 1$ and $u^{\prime}$ is Lipschitz in $\Omega^{\prime}$ (see for instance [9]).

Let $u_{n}=u^{\prime} \chi_{\Omega_{n}}$ for $n$ large. Clearly,

$$
\lim _{n \rightarrow+\infty} \int_{\Omega_{n}}\left|\nabla u_{n}\right| \mathrm{d} x=\lim _{n \rightarrow+\infty} \int_{\Omega_{n}}\left|\nabla u^{\prime}\right| \mathrm{d} x=\int_{\Omega}|\nabla u| \mathrm{d} x .
$$

By the assumption $\mathcal{H}^{N-1}\left(\partial A_{n}\right) \rightarrow \mathcal{H}^{N-1}(\partial A)$, we have, weakly-* as measures,

$$
\mathcal{H}^{N-1}\left\llcorner\partial A_{n} \rightarrow \mathcal{H}^{N-1}\llcorner\partial A,\right.
$$

where $\mathcal{H}^{N-1}\left\llcorner\partial A_{n}\right.$ and $\mathcal{H}^{N-1}\left\llcorner\partial A\right.$ denote the restrictions of the Hausdorff measure to $\partial A_{n}$ and $\partial A$ respectively. Moreover, $\sigma_{n} u^{\prime} \rightarrow \sigma u^{\prime}$ uniformly in $\Omega^{\prime}$. Hence,

$$
\int_{\partial A_{n}} \sigma_{n} u_{n} \mathrm{~d} \mathcal{H}^{N-1}=\int_{\partial A_{n}} \sigma_{n} u^{\prime} \mathrm{d} \mathcal{H}^{N-1} \rightarrow \int_{\partial A} \sigma u^{\prime} \mathrm{d} \mathcal{H}^{N-1}=\int_{\partial A} \sigma u \mathrm{~d} \mathcal{H}^{N-1} .
$$

We conclude that $\mathcal{E}_{n}\left(u_{n}\right) \rightarrow \mathcal{E}(u)$, i.e. (2.11) holds, and this finishes the proof.

\section{Numerical approximation}

In this section we provide an error estimate for the discretization of the problem discussed in Section 2 . We complete the analysis by describing the minimization algorithm that will be employed for the numerical simulations and introducing a finite element formulation of the equivalent convex problem (2.7).

\subsection{Error estimates}

We wish to compute $\bar{\sigma}$ in (2.4) as precisely as possible. We assume, for simplicity, that $\partial A$ is a polygonal boundary. By Proposition 2.8, any Lipschitz surface $\partial A$ can be replaced by a polygonal set with a small error, provided its total surface $\mathcal{H}^{N-1}(\partial A)$ is precisely approximated. However, the error obtained in this case can be tricky to estimate. The technique for obtaining error bounds for problems with discontinuous solution is relatively standard and consists first in smoothing the solution, it has been applied in particular for studying error bounds in Total Variation based regularization in $[13,18]$.

If $\partial A$ is polygonal we can find, for each $h>0$, a triangulation $\mathcal{T}_{h}$ of $\Omega$ such that each simplex $T$ of $\mathcal{T}_{h}$ has a diameter less than $h$. Moreover, we consider a standard regularity assumption on the triangulation, i.e., there exists a constant $K$, not dependent on $h$, such that the radius of the largest ball contained in each $T \in \mathcal{T}_{h}$ is more than the diameter of $T$, divided by $K$. 
The approximate problem is

$$
\bar{\sigma}_{h}=\min _{u \in V_{h}} \mathcal{E}(u)
$$

where $\mathcal{E}(u)$ is defined as in Proposition 2.8 and $V_{h}$ denotes the set of piecewise affine functions in $C(\Omega)$, affine on each $T \in T_{h}$, with value 1 on $\Gamma$.

Let $u=\chi_{E}$ be a solution of (2.4). Consider $u^{\delta} \in C^{2}(\bar{\Omega})$, with $u=1$ on $\Gamma$ and such that for some constant $C>0$,

$$
\left|D^{2} u^{\delta}\right| \leq \frac{C}{\delta^{2}} \text { and }\left\{u \neq u^{\delta}\right\} \subset\{\operatorname{dist}(\cdot, \partial E \cup \partial A) \leq C \delta\} .
$$

In particular, $u$ and $u^{\delta}$ are constant at some distance from $\partial E \cup \partial A$, which is a closed set of finite measure $\mathcal{H}^{N-1}$.

Let $u_{h}^{\delta}=\Pi_{h}\left(u^{\delta}\right)$ be the Lagrange interpolation of $u^{\delta}$ on $\mathcal{T}_{h}$, i.e., $u_{h}^{\delta} \in V_{h}$ and $u_{h}^{\delta}=u^{\delta}$ at each vertex of each simplex of $\mathcal{T}_{h}$. Standard interpolation arguments show that, for every $T \in \mathcal{T}_{h}$,

$$
\int_{T}\left|\nabla u_{h}^{\delta}-\nabla u^{\delta}\right| \mathrm{d} x \leq c|T| \frac{\operatorname{diam}(T)^{2}}{\rho(T)}\left\|D^{2} u^{\delta}\right\|_{L^{\infty}(T)} \leq c K|T| h \frac{C}{\delta^{2}},
$$

where $c$ is an explicit constant depending only on the dimension and $\operatorname{diam}(T), \rho(T)$ are respectively the diameter of $T$ and the radius of the largest ball contained in $T$. On the other hand, if $\Sigma$ is a facet of $T$,

$$
\int_{\Sigma}\left|u_{h}^{\delta}-u^{\delta}\right| \mathrm{d} \mathcal{H}^{N-1} \leq c|\Sigma| \operatorname{diam}(\Sigma)^{2}\left\|D^{2} u^{\delta}\right\|_{L^{\infty}(\Sigma)} \leq c|\Sigma| h^{2} \frac{C}{\delta^{2}}
$$

We deduce that

$$
\mathcal{E}\left(u_{h}^{\delta}\right) \leq \mathcal{E}\left(u^{\delta}\right)+c K\left|\left\{u \neq u_{h}^{\delta}\right\}\right| \frac{C h}{\delta^{2}}+c \mathcal{H}^{N-1}(\partial A) \frac{C h^{2}}{\delta^{2}} .
$$

Since $u_{h}^{\delta}=u^{\delta}=u$ at a distance from $\partial A \cup \partial E$ larger than $C \delta+h$, using (3.2) and (2.5), we get

$$
\left|\left\{u \neq u_{h}^{\delta}\right\}\right| \leq 4(C \delta+h) \mathcal{H}^{N-1}(\partial E \cup \partial A)
$$

if $C \delta+h$ is small enough. Assuming also $h \leq \delta$, we can find a constant (still denoted by $c$ ), depending on $K$, on the dimension, on $C$ and on the energy of $\chi_{E}$, such that

$$
\mathcal{E}\left(u_{h}^{\delta}\right) \leq \mathcal{E}\left(u^{\delta}\right)+c \frac{h}{\delta}
$$

Now suppose that we can build $u^{\delta}$ such that, for some positive constant (denoted again by $c$ ),

$$
\mathcal{E}\left(u^{\delta}\right) \leq \mathcal{E}(u)+c \delta=\bar{\sigma}+c \delta .
$$

Then, from (3.5) and (3.6), we deduce that, in order to minimize the global error, the optimal choice for $\delta$ is $\delta=\delta_{h} \simeq \sqrt{h}$. Letting $u_{h}=u_{h}^{\delta_{h}}$, we eventually get the error estimate

$$
\bar{\sigma} \leq \mathcal{E}\left(u_{h}\right) \leq \bar{\sigma}+c \sqrt{h}
$$

It is easy to build $u^{\delta}$ in a few situations. In dimension $N=2$, since $\partial A$ is piecewise affine, one can show that $\partial E \cap \Omega$ is a finite union of straight lines connecting two points of $\partial A$. In this case, we can find a small $\delta>0$ such that we can add a segment of length $\delta$ to both extremities of each of these lines, so that the segment is in the interior of $A$, except for its end which is in common with $\partial E$. This argument allows to extend the set $E$ to a set $E^{\delta}$ defined in the whole set $\{x \in Q: \operatorname{dist}(x, \Omega)<c \delta\}$ for some constant $c>0$, depending only on $\partial A$. We finally mollify $\chi_{E^{\delta}}$ by convolution with a radially symmetric kernel $\left(1 / \delta^{\prime N}\right) \eta\left(x / \delta^{\prime}\right)$, supported in the ball 
centered at the origin with radius $\delta^{\prime}=c \delta$. The result, restricted to $\Omega$, is a function $u^{\delta}$ satisfying both (3.2) and (3.6).

In higher dimension, the situation is more complicated. If we know, for instance, that there exists a constant $c$ such that

$$
\mathcal{H}^{N-1}(\partial E \cap\{0<\operatorname{dist}(x, \partial A)<\delta\}) \leq c \delta
$$

then by standard techniques we can reflect $E$ across $\partial A$ and the proof will follow as in dimension $N=2$. However, such estimate is not clear in general.

Remark 3.1. The error estimate (3.7) has been derived on simplices. However, for the numerical implementation, we found it more efficient to use unstructured quadrilateral meshes in dimension $N=2$ and hexaedral meshes in dimension $N=3$. There is no difficulty in extending the error estimate (3.7) to a set $\Omega=Q \backslash A$ which is approximated, for instance, by piecewise $N$-linear polynomials. We would get similar estimates for $u_{h}^{\delta}$ and then for $u_{h}$. But, in order to get rigorously all these estimates, we have to assume that the boundary $\partial A$ of the meshed domain is piecewise linear, fixed and not depending on the mesh size (which is even more restrictive with quadrilaterals than with simplices). In practice, our mesh also approximates a piecewise smooth boundary $\partial A$ (with an error $\mathcal{O}(h)$ on the surface). In this situation, it is quite difficult to extend the analysis into a sound error estimate, and would require a precise knowledge of the regularity of $u$ near $\partial A$. We expect that this does not change the final order of the approximation. Indeed, we found experimentally that the global order is much better than expected, of order $h$ rather than $h^{1 / 2}$ (see Fig. 8 in Sect. 4). Understanding this behaviour is the subject of future studies.

\subsection{The minimization scheme: $A D M M$}

From a numerical point of view, the major issue in finding the solution of the equivalent convex formulation of (2.4) is the boundedness requirement $u \geq 0$. The natural way to tackle this kind of minimization is to use splitting techniques among which the most natural would be the Douglas-Rachford splitting [7, 14], after a careful rewriting of the optimality conditions. Very useful for this kind of problem is also the so called ADMM ("Alternating Direction Method of Multipliers", also known as "split Bregman", see [8] for details) which is very flexible. After having investigated both directions, we have found the second one more efficient for this problem, and will now describe it. ADMM is a Lagrangian-based technique, which is very popular in image restoration problems, where a TV- $l_{1}$ minimization is needed.

Following [8], we give here a brief sketch of the method. Consider the problem

$$
\min _{\substack{u \in \mathbb{R}^{m} \\ K u=f}} J(u)
$$

and assume that $J(u)$ has separable structure, i.e. it can be written as

$$
J(u)=H(u)+\sum_{i=1}^{M} G_{i}\left(\widehat{A}_{i} u+b_{i}\right)
$$

where $H: \mathbb{R}^{m} \rightarrow(-\infty, \infty]$ and all the $G_{i}: \mathbb{R}^{n_{i}} \rightarrow(-\infty, \infty]$ are closed proper convex functions. Moreover, $f \in \mathbb{R}^{s}, b_{i} \in \mathbb{R}^{n_{i}}, \widehat{A}_{i}$ is a $n_{i} \times m$ matrix and $K$ is a $s \times m$ matrix.

Introducing new variables $p_{i}=\widehat{A}_{i} u+b_{i}$, we can rewrite $(3.8)$ as

$$
\min _{\substack{p \in \mathbb{R}^{n}, u \in \mathbb{R}^{m} \\ B p+\widehat{A} u+b=0}} F(p)+H(u),
$$

where

$$
F(p)=\sum_{i=1}^{M} G_{i}\left(p_{i}\right), \quad n=\sum_{i=1}^{M} n_{i}, \quad p=\left(p_{1}, \cdots, p_{M}\right)^{T}, \quad b=\left(b_{1}, \cdots, b_{M}, f\right)^{T}
$$


and

$$
B=\left[\begin{array}{c}
-I \\
0
\end{array}\right], \quad \widehat{A}=\left[\begin{array}{c}
\widehat{A}_{1} \\
\vdots \\
\widehat{A}_{M} \\
-K
\end{array}\right] .
$$

The augmented Lagrangian associated to the primal problem (3.9) is

$$
L_{\delta}(p, u, \mu)=F(p)+H(u)+\langle\mu, b-\widehat{A} u-B p\rangle+\frac{\delta}{2}\|b-\widehat{A} u-B p\|^{2},
$$

where the dual variable $\mu \in \mathbb{R}^{d}$ (with $d=n+s$ ) is interpreted as a vector of Lagrange multipliers. The dual functional $q_{\delta}: \mathbb{R}^{d} \rightarrow[-\infty, \infty)$ is a concave function defined by

$$
q_{\delta}(\mu)=\inf _{\substack{u \in \mathbb{R}^{m} \\ p \in \mathbb{R}^{n}}} L_{\delta}(p, u, \mu)
$$

The dual problem to (3.9) is then

$$
\max _{\mu \in \mathbb{R}^{d}} q_{\delta}(\mu) .
$$

Since (3.9) is a convex programming problem with linear constraints, if it has an optimal solution $\left(p^{*}, u^{*}\right)$, then (3.10) also has an optimal solution $\mu^{*}$ and

$$
F\left(p^{*}\right)+H\left(u^{*}\right)=q_{\delta}\left(\mu^{*}\right),
$$

which is to say that the duality gap is zero. It follows that finding an optimal solution to (3.9) and (3.10) is equivalent to finding a saddle point of $L_{\delta}$. More precisely, $\left(p^{*}, u^{*}\right)$ is an optimal primal solution and $\mu^{*}$ is an optimal dual solution if and only if

$$
L_{\delta}\left(p^{*}, u^{*}, \mu\right) \leq L_{\delta}\left(p^{*}, u^{*}, \mu^{*}\right) \leq L_{\delta}\left(p, u, \mu^{*}\right) \quad \forall p, u, \mu .
$$

Starting from an initial guess $u^{0}, \mu^{0}$, the iterations of the $A D M M$ algorithm at step $(k+1)$, with $k \in \mathbb{N}$, are given by

$$
\begin{aligned}
& p^{k+1}=\underset{p \in \mathbb{R}^{n}}{\operatorname{argmin}} L_{\delta}\left(p, u^{k}, \mu^{k}\right), \\
& u^{k+1}=\underset{u \in \mathbb{R}^{m}}{\operatorname{argmin}} L_{\delta}\left(p^{k+1}, u, \mu^{k}\right), \\
& \mu^{k+1}=\mu^{k}+\delta\left(b-\widehat{A} u^{k+1}-B p^{k+1}\right) .
\end{aligned}
$$

\subsection{Finite element discretization}

For $h>0$, let $\mathcal{T}_{h}$ be an unstructured subdivision of $\Omega$ into quadrilaterals (in dimension $N=2$, hexaedra in dimension $N=3$ ) of maximal diameter $h$ and let $K_{l}$ be the $l$-th element of the corresponding mesh. Besides, let $\partial A_{h}$ be a polygonal approximation of the boundary of the solid $A$. In order to simplify the presentation, we identify, with a little abuse of notation, $\mathcal{T}_{h}$ with $\Omega$ and $\partial A_{h}$ with $\partial A$. We then consider two finite dimensional spaces $V_{h}$ and $W_{h}$ for the discrete representation of $u$ and $D u$ respectively, by means of piecewise $N$-linear and piecewise constant polynomials:

$$
\begin{aligned}
V_{h} & =\left\{v \in C^{0}(\Omega):\left.v\right|_{K_{l}} \in \mathcal{Q}^{1}, l=1, . ., N_{e}\right\}, \\
W_{h} & =\left\{w \in L^{2}(\Omega)^{N}:\left.w\right|_{K_{l}} \in\left[\mathcal{P}^{0}\right]^{N}, l=1, . ., N_{e}\right\},
\end{aligned}
$$


where $N_{e}$ is the number of elements of the mesh, $\mathcal{Q}^{1}$ denotes the polynomials of degree 1 in each coordinate direction and $\left[\mathcal{P}^{0}\right]^{N}$ denotes the $N$-dimensional vectors of constant polynomials. In the Finite Element terminology this choice is known as the $Q 1-P 0$ approximation. We remark that the reference basis for $\mathcal{Q}^{1}$ is given, in dimension $N=2$, by $\{1, x, y, x y\}$ and that the degrees of freedom of $V_{h}$ are identified by the vertices of the quadrilaterals (hexaedra in dimension $N=3$ ). On the other hand, the space $W_{h}$ contains discontinuous functions and its degrees of freedom are located at the baricenters of the elements.

In the discretized form, problem (2.7) can be read as

$$
\bar{\sigma}_{2}=\min \left\{\int_{\Omega}|\nabla u|+\int_{\partial A} \sigma|u|: u \in V_{h}, u=1 \text { on } \Gamma\right\} .
$$

Note that, for a discrete function $u \in V_{h}$, the total variation term $\int_{\Omega}|D u|$ reduces to the $L^{1}$ norm of its gradient.

Now we can write the augmented Lagrangian associated with the energy in (3.12). We introduce $p_{1} \in W_{h}$ and $p_{2} \in V_{h}$ as auxiliary variables for the two terms in the functional and we denote by $n_{1}$ the number of degrees of freedom (dofs for short) of $W_{h}$, by $n_{2}$ the number of dofs of $V_{h}$. Moreover, let us identify discrete functions in $W_{h}$ and $V_{h}$ by the vectors of their dofs in the finite element representation, so that we can also write, with a little abuse of notation, $p_{1} \in \mathbb{R}^{n_{1}}$ and $p_{2} \in \mathbb{R}^{n_{2}}$ (see Sect. 3.4 for further details). According to the notation of the previous section, we set

$$
\begin{gathered}
K=0, \quad f=0, \quad H=0, \quad n=n_{1}+n_{2}, \quad p=\left(p_{1}, p_{2}\right)^{T} \in \mathbb{R}^{n}, \quad b=(0,0)^{T}, \\
F(p)=F\left(p_{1}, p_{2}\right)=G_{1}\left(p_{1}\right)+G_{2}\left(p_{2}\right)=\int_{\Omega}\left|p_{1}\right|+\int_{\partial A} \sigma\left|p_{2}\right|, \\
\mu=\left(\mu_{1}, \mu_{2}\right) \in \mathbb{R}^{n}, \quad B=-I, \quad \widehat{A}=\left[\begin{array}{c}
\nabla \\
I
\end{array}\right]
\end{gathered}
$$

where $I$ denotes the identity operator and $\nabla$ is a discrete version of the gradient operator, that will be detailed in Section 3.4. Then we can write, for $\delta>0$,

$$
\begin{aligned}
L_{\delta}(p, u, \mu)= & \int_{\Omega}\left|p_{1}\right|+\int_{\Omega} \mu_{1} \cdot\left(p_{1}-\nabla u\right)+\frac{\delta}{2} \int_{\Omega}\left|p_{1}-\nabla u\right|^{2} \\
& +\int_{\partial A} \sigma\left|p_{2}\right|+\int_{\partial A} \mu_{2}\left(p_{2}-u\right)+\frac{\delta}{2} \int_{\partial A}\left|p_{2}-u\right|^{2} .
\end{aligned}
$$

Following the ADMM algorithm, we define the alternating steps in (3.11) for every $k \in \mathbb{N}$. We minimize first in $p$, i.e. we find, given the initial guess $\mu^{0}=0$ and $u^{0}$ arbitrary,

$$
\begin{aligned}
p^{k+1}=\underset{p \in \mathbb{R}^{n}}{\operatorname{argmin}} \int_{\Omega}\left|p_{1}\right|+\int_{\Omega} \mu_{1}^{k} \cdot\left(p_{1}-\nabla u^{k}\right)+\frac{\delta}{2} \int_{\Omega}\left|p_{1}-\nabla u^{k}\right|^{2} \\
+\int_{\partial A} \sigma\left|p_{2}\right|+\int_{\partial A} \mu_{2}^{k}\left(p_{2}-u^{k}\right)+\frac{\delta}{2} \int_{\partial A}\left|p_{2}-u^{k}\right|^{2} .
\end{aligned}
$$

This is equivalent to finding

$$
p^{k+1}=\underset{p \in \mathbb{R}^{n}}{\operatorname{argmin}} \int_{\Omega}\left[\left|p_{1}\right|+\frac{\delta}{2}\left|p_{1}-\nabla u^{k}+\frac{\mu_{1}^{k}}{\delta}\right|^{2}\right]+\int_{\partial A}\left[\sigma\left|p_{2}\right|+\frac{\delta}{2}\left|p_{2}-u^{k}+\frac{\mu_{2}^{k}}{\delta}\right|^{2}\right] .
$$

Then, we can split the global minimization for $p$ into two sub-problems for $p_{1}$ and $p_{2}$ and argue that, with little abuse of notation,

$$
\begin{aligned}
p^{k+1} & =\underset{p \in \mathbb{R}^{n}}{\operatorname{argmin}} L_{\delta}\left(p, u^{k}, \mu^{k}\right)=\left(\underset{p_{1} \in \mathbb{R}^{n_{1}}}{\operatorname{argmin}} L_{\delta}\left(p, u^{k}, \mu^{k}\right), \underset{p_{2} \in \mathbb{R}^{n_{2}}}{\operatorname{argmin}} L_{\delta}\left(p, u^{k}, \mu^{k}\right)\right) \\
& =\left(p_{1}^{k+1}, p_{2}^{k+1}\right) .
\end{aligned}
$$


We look at the first term in (3.14) and define $a=\nabla u^{k}-\frac{\mu_{1}^{k}}{\delta}$. We get the following chain of inequalities:

$$
\begin{aligned}
T\left(p_{1}\right):=\int_{\Omega}\left[\left|p_{1}\right|+\frac{\delta}{2}\left|p_{1}-a\right|^{2}\right] & =\int_{\Omega}\left[\left|p_{1}\right|+\frac{\delta}{2}\left|p_{1}\right|^{2}-\delta\left(p_{1} \cdot a\right)+\frac{\delta}{2}|a|^{2}\right] \\
& \geq \int_{\Omega}\left[\left|p_{1}\right|+\frac{\delta}{2}\left|p_{1}\right|^{2}-\delta\left|p_{1}\right||a|+\frac{\delta}{2}|a|^{2}\right] \\
& \geq \int_{\Omega}\left[\frac{\delta}{2}|a|^{2}+\left|p_{1}\right|(1-\delta|a|)\right] .
\end{aligned}
$$

Now, if $(1-\delta|a|) \geq 0$, we conclude that $T\left(p_{1}\right) \geq \int_{\Omega} \frac{\delta}{2}|a|^{2}$ for every $p_{1} \in \mathbb{R}^{n_{1}}$ and the minimum value is attained at $p_{1}=0$. On the other hand, If $(1-\delta|a|)<0$, we can compute the differential of $T$ out of the origin,

$$
\nabla T\left(p_{1}\right)=\int_{\Omega}\left[\frac{p_{1}}{\left|p_{1}\right|}+\delta\left(p_{1}-a\right)\right]
$$

which is equal to 0 for $p_{1}=\frac{a}{|a|}\left(|a|-\frac{1}{\delta}\right)$ and we conclude that

$$
p_{1}^{k+1}=\left(1-\frac{1}{\max (1, \delta|a|)}\right) a .
$$

More generally, we can introduce the shrinkage operator $\mathcal{S}_{\lambda}: v \longmapsto \frac{v}{|v|}(|v|-\lambda)^{+}$, so that

$$
p_{1}^{k+1}=\mathcal{S}_{\frac{1}{\delta}}\left(\nabla u^{k}-\frac{\mu_{1}^{k}}{\delta}\right) .
$$

Similarly, minimizing (3.14) in $p_{2}$, we get

$$
p_{2}^{k+1}=\mathcal{S}_{\frac{\sigma}{\delta}}\left(u^{k}-\frac{\mu_{2}^{k}}{\delta}\right) .
$$

The next step is the minimization in $u$, i.e., finding

$$
u^{k+1}=\underset{u \in \mathbb{R}^{n_{2}}}{\operatorname{argmin}} L_{\delta}\left(p^{k+1}, u, \mu^{k}\right) .
$$

By omitting in $L_{\delta}\left(p^{k+1}, u, \mu^{k}\right)$ the terms containing only $p_{1}^{k+1}, p_{2}^{k+1}, \mu_{1}^{k}$ and $\mu_{2}^{k}$, which are constant with respect to $u$, we have

$$
\begin{aligned}
u^{k+1}= & \underset{u \in \mathbb{R}^{n_{2}}}{\operatorname{argmin}} \frac{\delta}{2} \int_{\Omega}\left[|\nabla u|^{2}-2\left(p_{1}^{k+1}+\frac{\mu_{1}^{k}}{\delta}\right) \cdot \nabla u\right] \\
& +\frac{\delta}{2} \int_{\partial A}\left[|u|^{2}-2\left(p_{2}^{k+1}+\frac{\mu_{2}^{k}}{\delta}\right) u\right] .
\end{aligned}
$$

Let us define $c_{1}^{k}:=p_{1}^{k+1}+\frac{\mu_{1}^{k}}{\delta}$ and $c_{2}^{k}:=p_{2}^{k+1}+\frac{\mu_{2}^{k}}{\delta}$. Then, the stationarity condition for (3.18) reads, for every $v \in V_{h}$ such that $v \mid \Gamma=0$, as

$$
\int_{\Omega} \nabla u \cdot \nabla v+\int_{\partial A} u v=\int_{\Omega} c_{1}^{k} \cdot \nabla v+\int_{\partial A} c_{2}^{k} v
$$


As we will see in the next section, (3.19) gives rise to a linear system, whose solution $u^{k+1}$ converges, as $k \rightarrow+\infty$, to the numerical solution of our problem. Moreover, the boundary condition $u=1$ on $\Gamma$ will be imposed directly on the linear system, by means of a Gaussian elimination.

The last step consists in the update of the Lagrange multipliers, which is done in a standard way:

$$
\begin{aligned}
& \mu_{1}^{k+1}=\mu_{1}^{k}+\delta\left(p_{1}^{k+1}-\nabla u^{k+1}\right), \\
& \mu_{2}^{k+1}=\mu_{2}^{k}+\delta\left(p_{2}^{k+1}-u^{k+1}\right) .
\end{aligned}
$$

\subsection{Numerical implementation}

The numerical implementation of the ADMM algorithm for our problem involves three main steps: the representation of $\nabla u$, the representation of $p$, i.e. the numerical counterpart of the shrinkage operator, and the representation of $u$, i.e. the solution of the linear system associated to the discretization of the elliptic equation (3.19).

Step 1. Since $u$ and $\nabla u$ are defined on different finite element spaces, each one with its own degrees of freedom and set of basis functions, we have to find a representation of $\nabla u$ in $W_{h}$ for a given $u$ in $V_{h}$. So, given $u=\sum_{i=1}^{n_{2}} u_{i} \phi_{i}$, where $\left\{\phi_{i}\right\}$ is a basis for $V_{h}$ and $\left\{u_{i}\right\} \in \mathbb{R}^{n_{2}}$, we represent $\nabla u$ through the projection $\Pi(\nabla u)$ onto $W_{h}$. Let $\left\{\psi_{j}\right\}$ be a basis for $W_{h}$ and cosider the problem of finding $\left\{w_{j}\right\} \in \mathbb{R}^{n_{1}}$ such that $\Pi(\nabla u)=\sum_{j=1}^{n_{1}} w_{j} \psi_{j}$ pointwise, i.e., for every $x \in \Omega$,

$$
\sum_{j=1}^{n_{1}} w_{j} \psi_{j}(x)=\sum_{i=1}^{n_{2}} u_{i} \nabla \phi_{i}(x) .
$$

We take the scalar product between each side of the equation and $\psi_{k}(x)$, we perform a summation over $k=$ $1, \ldots, n_{1}$ and we integrate on $\Omega$, so that

$$
\sum_{k=1}^{n_{1}} \sum_{j=1}^{n_{1}} w_{j} \int_{\Omega} \psi_{j}(x) \cdot \psi_{k}(x)=\sum_{k=1}^{n_{1}} \sum_{i=1}^{n_{2}} u_{i} \int_{\Omega} \nabla \phi_{i}(x) \cdot \psi_{k}(x) .
$$

Then, we have to solve the following linear system:

$$
\mathbf{M}^{\psi} \mathbf{w}=\mathbf{T}^{\nabla \phi, \psi} \mathbf{u}
$$

where $\mathbf{M}^{\psi}$ is the mass matrix of $W_{h}, \mathbf{w}=\left\{w_{j}\right\}$ is the unknown vector representing $\nabla u$ on $W_{h}, \mathbf{T}^{\nabla \phi, \psi}$ is the $n_{1} \times n_{2}$ matrix for the change of coordinates between $V_{h}$ and $W_{h}$ and $\mathbf{u}=\left\{u_{i}\right\}$ is the vector representing $u$ on $V_{h}$.

Step 2. The second issue of our implementation is the shrinkage operator related to the minimization of the augmented Lagrangian (3.13) with respect to $p_{1} \in W_{h}$ and $p_{2} \in V_{h}$. As in Step 1, we need to project the pointwise formulas (3.16) and (3.17) on $W_{h}$ and $V_{h}$ respectively.

Recalling the definition $a=\nabla u-\frac{\mu_{1}}{\delta}$, represented on $W_{h}$ by $\left\{a_{j}\right\} \in \mathbb{R}^{n_{1}}$, we get for $p_{1}$

$$
\sum_{j=1}^{n_{1}} p_{1, j} \psi_{j}(x)=\left(1-\frac{1}{\max \left(1, \delta\left|\sum_{l=1}^{n_{1}} a_{l} \psi_{l}(x)\right|\right)}\right) \sum_{j=1}^{n_{1}} a_{j} \psi_{j}(x),
$$

from which we obtain the following linear system:

$$
\mathbf{M}^{\psi} \mathbf{p}_{\mathbf{1}}=\mathbf{S}_{\frac{1}{\delta}}^{\psi}[\mathbf{a}] \mathbf{a}
$$




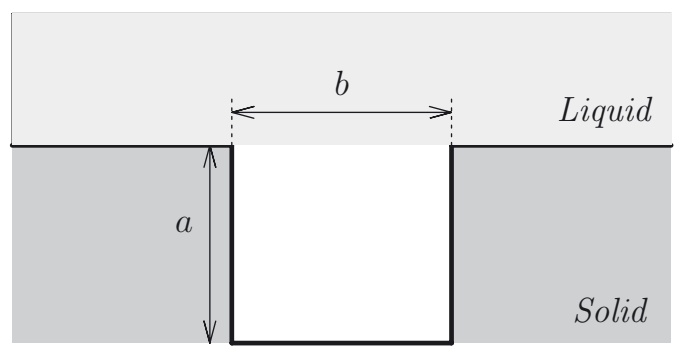

(a)

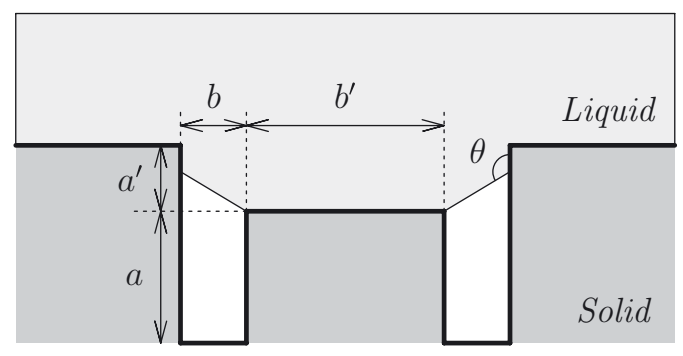

(b)

FiguRE 6. Benchmark configurations. (a) Pillars with the same height. (b) A particular geometry leading to an intermediate equilibrium between Wenzel and Cassie-Baxter configurations.

where $\mathbf{M}^{\psi}$ is the mass matrix of $W_{h}, \mathbf{p}_{\mathbf{1}}$ is the unknown vector representing $p_{1}$ on $W_{h}$, a is the vector representing $a$ on $W_{h}$ and $\mathbf{S}_{\frac{1}{\delta}}^{\psi}[\mathbf{a}]$ is the $n_{1} \times n_{1}$ shrinkage matrix defined by

$$
\left(\mathbf{S}_{\frac{1}{\delta}}^{\psi}[\mathbf{a}]\right)_{j k}=\int_{\Omega}\left(1-\frac{1}{\max \left(1, \delta\left|\sum_{l=1}^{n_{1}} \widehat{a_{l}} \psi_{l}(x)\right|\right)}\right) \psi_{j}(x) \cdot \psi_{k}(x) .
$$

Similarly, for $a=u-\frac{\mu_{2}}{\delta}$ in $V_{h}$, we get the following linear system for $p_{2}$

$$
\mathbf{M}^{\phi} \mathbf{p}_{2}=\mathbf{S}_{\frac{\sigma}{\delta}}^{\phi}[\mathbf{a}] \mathbf{a}
$$

with analogous meaning of the symbols.

Step 3. The last step, concerning the minimization with respect to $u$, is standard. By classical arguments, it is easy to see that (3.19) defines a bilinear form $\mathcal{L}: V_{h} \times V_{h} \rightarrow \mathbb{R}$ and a linear continuous functional $\mathcal{F}: V_{h} \rightarrow \mathbb{R}$, i.e.,

$$
\begin{aligned}
\mathcal{L}(u, v) & :=\int_{\Omega} \nabla u(x) \cdot \nabla v(x)+\int_{\partial A} u(x) v(x), \\
\mathcal{F}(v) & :=\int_{\Omega} c_{1}(x) \cdot \nabla v(x)+\int_{\partial A} c_{2}(x) v(x) .
\end{aligned}
$$

Solving the associated linear system completes the iterative scheme. We recall that the Dirichlet boundary condition $u=1$ on $\Gamma$ is enforced directly in the matrix of the linear system, by means of a standard Gaussian elimination.

\section{Numerical tests}

We begin by showing some benchmark experiments in dimension $N=2$, in order to check the performance of the method. We first consider configurations in which the minimal energy $\bar{\sigma}$, defining the cosine of the effective contact angle $\theta^{\text {hom }}$ of the homogenized surface, can be plainly computed and compared with our numerical results. In particular, for the case of equally spaced pillars with the same height, shown in Figure 6a, we would like to give an estimate of the critical surface tension $\sigma$ above which the liquid phase does not fill the space between the asperities.

Indeed, it is easy to see that such a geometry allows only for two possible minimizers: the first fills the cavities with the fluid (Wenzel configuration), the second performs the transition at the level of the top of the 
TABLE 1. Numerical results for the first benchmark test (see Fig. 6a).

\begin{tabular}{|c|c|c|c|c|c|}
\hline$\sigma$ & $\theta$ & Behaviour & $\bar{\sigma}$ & $\bar{\sigma}^{\text {num }}$ & $\theta^{\text {hom,num }}$ \\
\hline 0.32 & $108^{\circ}$ & Wenzel & 0.5333 & 0.5333 & $122^{\circ}$ \\
\hline 0.34 & $110^{\circ}$ & Cassie-Baxter & 0.56 & 0.5619 & $124^{\circ}$ \\
\hline
\end{tabular}

TABLE 2. Numerical results for the second benchmark test (see Fig. 6b).

\begin{tabular}{|c|c|c|c|c|c|}
\hline$\sigma$ & $\theta$ & Behaviour & $\bar{\sigma}$ & $\bar{\sigma}^{\text {num }}$ & $\theta^{\text {hom,num }}$ \\
\hline 0.2 & $101.5^{\circ}$ & Wenzel & 0.44 & 0.44 & $116^{\circ}$ \\
\hline 0.205 & $102^{\circ}$ & Mixed & 0.4417 & 0.4435 & $116^{\circ}$ \\
\hline 0.455 & $117^{\circ}$ & Mixed & 0.7241 & 0.7256 & $136.5^{\circ}$ \\
\hline 0.46 & $117^{\circ}$ & Cassie-Baxter & 0.73 & 0.7316 & $137^{\circ}$ \\
\hline
\end{tabular}

pillars (Cassie-Baxter configuration). So, if we choose $a=0.3333$ and $b=0.3334$, the critical $\sigma$ that establishes the switch is given by $\sigma_{\text {crit }}=0.3334$. We denote by the superscript "num" the quantities that are numerical approximations of the corresponding mathematical quantities. The numerical results are very satisfactory: we obtain the estimate $\sigma_{\text {crit }}^{\text {num }} \approx 0.335$.

Table 1 shows the results for different values of $\sigma$. We should specify that the accuracy of the approximation depends on the mesh size: exploiting adaptive mesh refinement techniques and obviously more computing resources, we can reach even much better estimates.

In the second test (see Fig. 6b) the possible behavior is more complicated, indeed it is allowed an equilibrium configuration of the liquid that combines features of both Wenzel (complete contact on tall asperities) and Cassie-Baxter (composite contact on short asperities) configurations. It is easy to show that this intermediate configuration is the optimal one if

$$
\frac{a^{\prime}}{b^{\prime}} \leq \frac{1-\sigma}{2 \sigma} \leq \frac{a}{b}
$$

By choosing $a=0.2, b=0.1, a^{\prime}=0.2$ and $b^{\prime}=0.3$, we obtain that the bounds in (4.1) are violated for $\sigma_{\text {crit }}^{\min }=0.2$ and $\sigma_{\text {crit }}^{\max }=0.4285$ respectively. To be precise, $\sigma_{\text {crit }}^{\max }$ is an approximation, since the corresponding bound in (4.1) has been obtained by replacing the length of the slightly tilted liquid-vapor interface with the length of its horizontal projection (see [1] for details).

For $\sigma \leq \sigma_{\text {crit }}^{\min }$ the liquid fills all the cavities (Wenzel configuration), whereas for $\sigma \geq \sigma_{\text {crit }}^{\max }$ it touches only the tallest faces of the pillars (Cassie-Baxter configuration). Also for this test the numerical results are very satisfactory: we obtain the estimates $\sigma_{\text {crit }}^{\text {min,num }} \approx 0.2$ and $\sigma_{\text {crit }}^{\text {max,num }} \approx 0.455$ (as mentioned above, the analytical critical values are affected by a simplification).

Figure 7 and Table 2 show, respectively, the numerical approximation of two minimizers and the results for different values of $\sigma$.

The two examples above can be also used for checking the error estimate (3.7) on uniform grids with square cells. Indeed, the local match of Young's law allows for the calculation of the exact $\bar{\sigma}$ in both cases. We have that the lower bound in (3.7) is always fulfilled, that is, given a mesh size $h$, we have that $\bar{\sigma}_{h}$ (the approximation of $\bar{\sigma}$ on $V_{h}$ ) is always greater than $\bar{\sigma}$. Unexpectedly, the upper bound behavior is even linear with respect to $h$ (see Fig. 8 and also Rem. 3.1).

In the case of pillars at the same height the analysis is straightforward. Indeed, the best approximation on $V_{h}$ of the exact solution $u$ is a function $u_{h}$ that makes a continuous transition between 0 to 1 along a straight array of cells $\left\{K_{l}\right\}$, placed at the same height of the pillars and just above the cavity, with $l=1, \ldots, n_{h}$ (up to a renumeration of the dofs) and $n_{h}=\frac{b}{h}$ (see Fig. 9 and also Fig. 6a). 

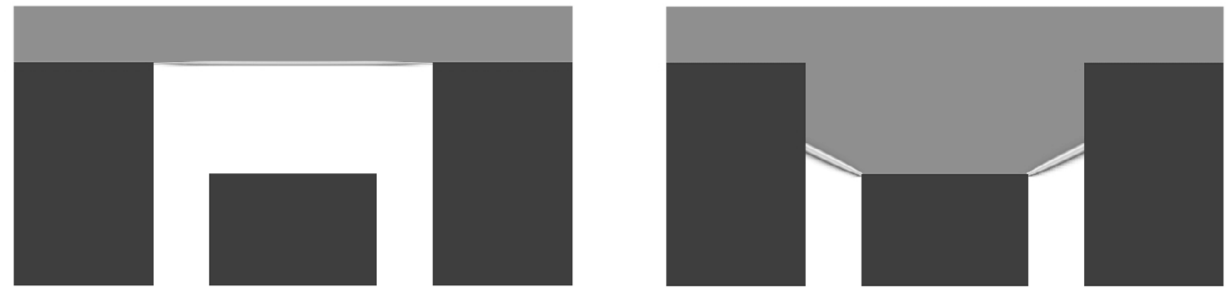

FiguRE 7. Visualization of two minimizers.

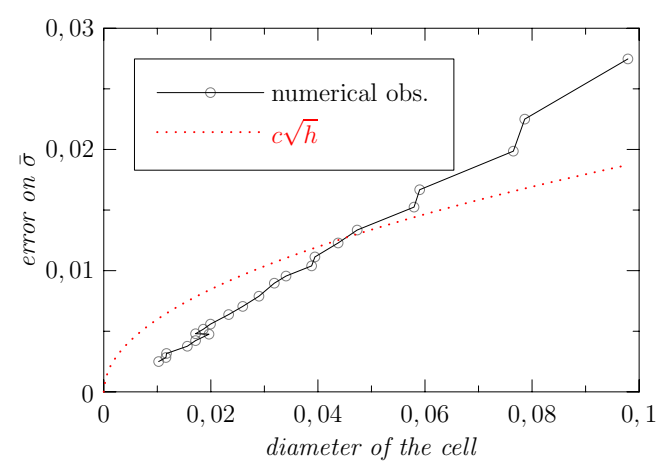

(a)

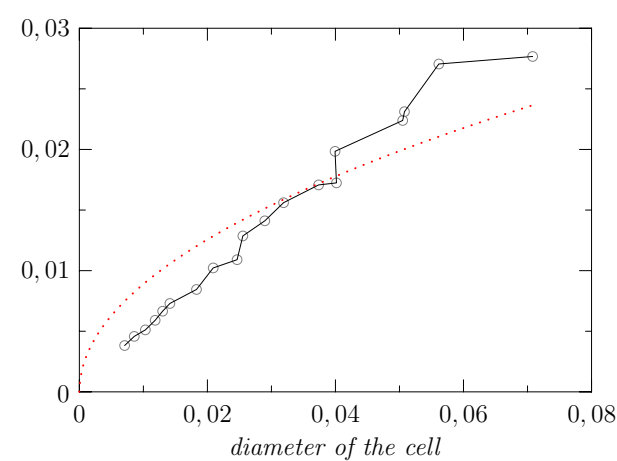

(b)

Figure 8. Plot of the error behaviour for the examples (a) and (b) in Figure 6. The multiplicative constant $c$ is the one that best fits the observations.

For these cells, the value of $u_{h}$ in the upper vertices is 1 and the value in the lower ones is 0 , so that, up to a translation, we have $\left.u_{h}(x, y)\right|_{K_{l}}=(1 / h) y$ for every $l=1, \ldots, n_{h}$ and

$$
\int_{\Omega}\left|\nabla u_{h}\right|=\sum_{l=1}^{n_{h}} \frac{1}{h}\left|K_{l}\right|=n_{h} \frac{1}{h} h^{2}=\frac{b}{h} h=b=\int_{\Omega}|D u|,
$$

that is the error due to the Total Variation term is 0 . Then, the error only depends on the tension term, in particular it is concentrated on the two vertical external edges of the cells with faces on the lateral walls of the cavity, and we get

$$
\mathcal{E}\left(u_{h}\right)-\mathcal{E}(u)=2 \sigma \frac{1}{h} \int_{0}^{h} y \mathrm{~d} y=\sigma h,
$$

where $\mathcal{E}$ still denotes the energy defined in Proposition 2.8. We conclude that, in this particular case, we not only can provide the analytical value of $\bar{\sigma}$, but we can also calculate the smallest possible error of $\bar{\sigma}_{h}$ on $V_{h}$. Numerical experiments show that the $A D M M$ algorithm is able to match the estimate (4.2), namely it can provide the best possible numerical approximation.

In the case of pillars at different heights, where the interface is tilted, the Total Variation term of the approximate solution cannot be directly computed. However, an analysis similar to the previous one still confirms that the error given by the tension term is $\mathcal{O}(h)$ and the total numerical error is $\mathcal{O}(h)$ as well (see Fig. 8b).

The third test deals with a solid surface of complex geometry. The choice of optimal configurations is no longer in a discrete set, as in the case of the first test, where only two equilibrium configurations are allowed. Here we have a continuous range of liquid-vapor interfaces, determined by the value of $\sigma$. 
TABLE 3. Numerical results for the test with a solid of complex geometry.

\begin{tabular}{|c|c|c|c|c|}
\hline$\sigma$ & $\theta$ & Behaviour & $\bar{\sigma}^{\text {num }}$ & $\theta^{\text {hom,num }}$ \\
\hline 0.2 & $101.5^{\circ}$ & Wenzel & 0.3307 & $109^{\circ}$ \\
\hline 0.45 & $116.7^{\circ}$ & Mixed & 0.7365 & $137.5^{\circ}$ \\
\hline 0.65 & $130.5^{\circ}$ & Mixed & 0.9203 & $157^{\circ}$ \\
\hline 0.82 & $148^{\circ}$ & Cassie-Baxter & 0.9884 & $171^{\circ}$ \\
\hline
\end{tabular}

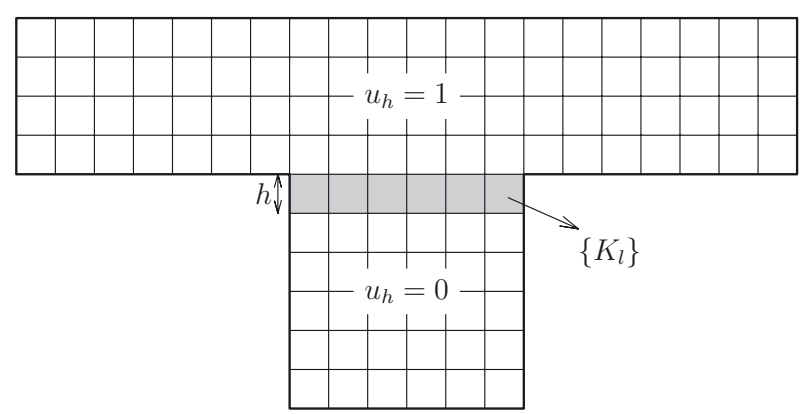

Figure 9. Best numerical approximation of the Cassie-Baxter configuration for pillars at the same height.

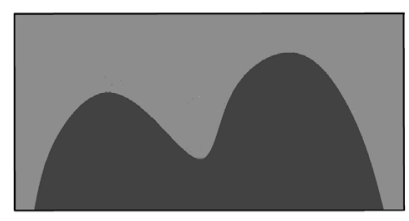

$$
\sigma=0.2
$$

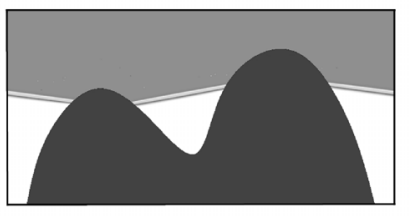

$\sigma=0.65$

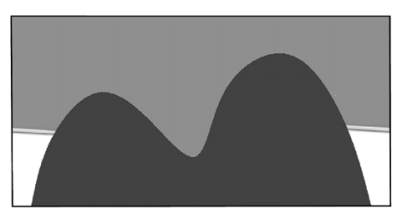

$\sigma=0.45$

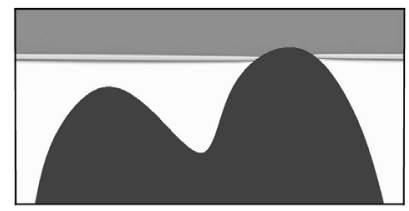

$\sigma=0.82$

FIGURE 10. Equilibrium configurations (depending on $\sigma$ ) on a solid of complex geometry.

Table 3 shows the numerical results, Figure 10 shows the corresponding equilibrium configurations as $\sigma$ increases.

We remark that in this case the periodic condition at the lateral boundary of the cell is not "naturally" matched as before. Indeed, in the previous tests, the position and the same height of the pillars forced the solution to be periodic. Here the periodicity of the solution is an actual constraint, hidden in the implementation of our finite element approximation. As a consequence, the liquid-vapor interfaces do not lie, in general, on straight lines, but have to follow diagonal trajectories.

For this set of simulations we also introduced a gradient detector refinement method, in order to refine the mesh only in the regions affecting the accuracy of the approximation, namely in small neighborhoods of the liquid-vapor interfaces (see Fig. 11). 


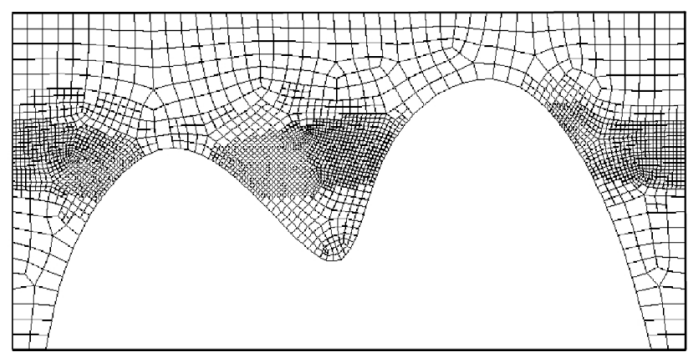

Figure 11. Adaptive mesh refinement.

TABLE 4. Numerical results for the 3D simulations.

\begin{tabular}{|c|c|c|c|c|c|}
\hline$\sigma$ & $\theta$ & Pillars type & Behaviour & $\bar{\sigma}^{\text {num }}$ & $\theta^{\text {hom,num }}$ \\
\hline 0.42 & $115^{\circ}$ & Cylindrical & Mixed & 0.8092 & $144^{\circ}$ \\
\hline 0.42 & $115^{\circ}$ & Square & Mixed & 0.761 & $139.5^{\circ}$ \\
\hline
\end{tabular}

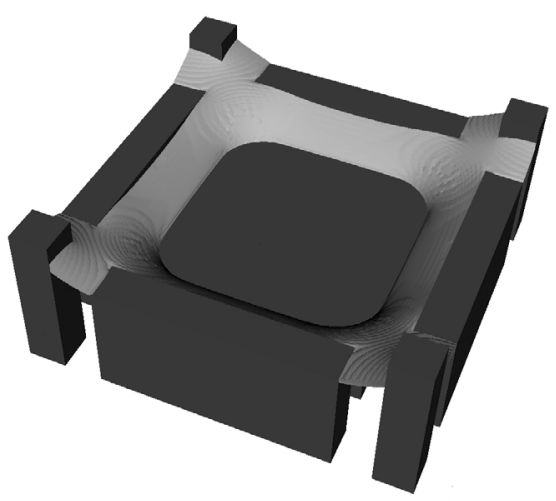

(a)

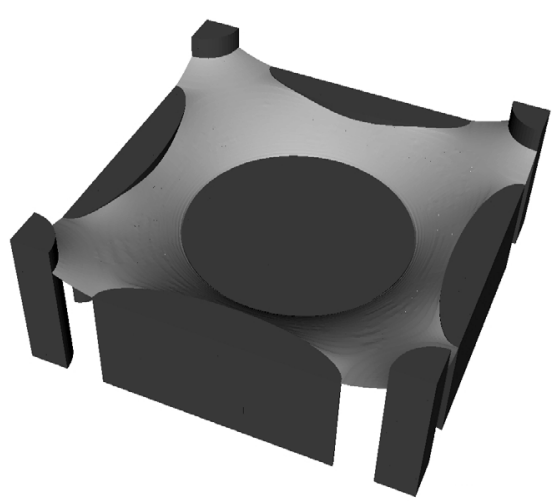

(b)

FIgURE 12. Equilibrium configurations on square (a) and cylindrical (b) pillars.

We proceed with some experiments in dimension $N=3$. Our aim is to recover, also in a three dimensional environment, the configurations of intermediate type observed in the previous tests. In this case, the liquid-vapor interfaces are no longer portions of straight lines, but are allowed to also assume bended shapes, making the analytical computation much more complicated.

We consider both square and cylindrical pillars at different height, whose coordinate sections exibit the peculiar sizes that, in two dimensions, let emerge the mixed configurations (neither Wenzel nor Cassie-Baxter).

Table 4 shows the numerical results for this test, Figure 12 shows the two type of pillars and also the isosurfaces, at level 0.5 , of the corresponding solutions.

Also in this setting there is a set of optimal solutions that can both touch the lateral part of the pillars and make the liquid-vapor transition. We remark that the boundary $\partial E$ of the geometrical solution $E$ (remember that our numerical solution is indeed an approximation of $\chi_{E}$ in (2.4)) is a minimal surface: the change of 


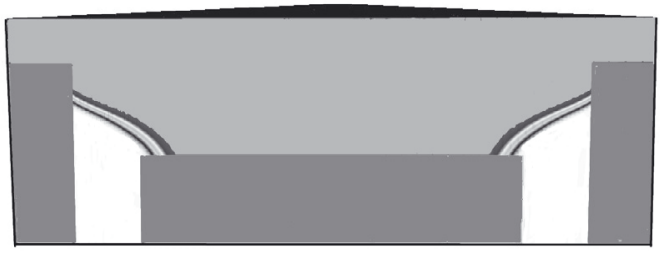

(a)

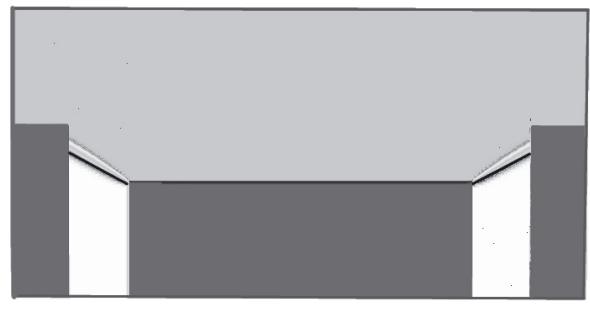

(b)

FIgURE 13. Diagonal (a) and horizontal (b) sections of the solution on square pillars.

curvature near the asperities is evident, especially in the case of square pillars. In particular, Figure 13a shows a diagonal section of the solution in which it is easy to see that the transition region is bended. On the other hand, slices of the solution along the coordinate axis, as the one shown in Figure 13b, exhibit the same behavior of the two dimensional experiments previously discussed.

Acknowledgements. The authors thank François Alouges and Luca Heltai for stimulating discussions. The fourth author is grateful to the SISSA in Trieste, where he was a Ph.D. student when this work was done. We also are very grateful to the anonymous reviewers who made precise and very helpful comments on the first version of this work. Part of this research was funded by the ANR "MICA", grant number ANR-08-BLAN-0082. Finally, a special acknowledgement goes to the open source $\mathrm{C}++$ finite element library deal.II (www.dealii.org), without which the simulations contained in this paper would not have been (almost) so easily realized.

\section{REFERENCES}

[1] G. Alberti and A. DeSimone, Wetting of rough surfaces: a homogenization approach. Proc. R. Soc. A 461 (2005) $79-97$.

[2] G. Alberti and A. DeSimone, Quasistatic evolution of sessile drops and contact angle hysteresis. Arch. Rat. Mech. Anal. 202 (2011) 295-348.

[3] L. Ambrosio, N. Fusco and D. Pallara, Functions of bounded variation and free discontinuity problems, Oxford Mathematical Monographs, The Clarendon Press Oxford University Press, New York (2000)

[4] L.A. Caffarelli, The obstacle problem revisited. J. Fourier Anal. Appl. 4 (1998) 383-402.

[5] L.A. Caffarelli and A. Mellet, Capillary drops on an inhomogeneous surface, Perspectives in nonlinear partial differential equations. Contemp. Math. 446 (2007) 175-201.

[6] A. DeSimone, N. Grunewald and F. Otto, A new model for contact angle hysteresis. Netw. Heterog. Media 2 (2007) $211-225$.

[7] J. Eckstein and D.P. Bertsekas, On the Douglas-Rachford splitting method and the proximal point algorithm for maximal monotone operators. Math. Program. 55 (1992)

[8] E. Esser, Applications of Lagrangian-Based Alternating Direction Methods and Connections to Split Bregman (2009)

[9] L.C. Evans and R.F. Gariepy, Measure theory and fine properties of functions. CRC Press, Boca Raton, FL (1992).

[10] L. Fedeli, A. Turco and A. DeSimone, Metastable equilibria of capillary drops on solid surfaces: a phase field approach. Contin. Mech. Thermodyn. 23 (2011) 453-471.

[11] H. Federer, Geometric measure theory. Springer-Verlag New York Inc., New York (1969).

[12] R. Finn, Some properties of capillary surfaces. Milan J. Math. 70 (2002) 1-23.

[13] M.J. Lai, B. Lucier and J. Wang, The convergence of a central-difference discretization of Rudin-Osher-Fatemi model for image denoising, Scale Space and Variational Methods in Computer Vision. Springer (2009) 514-526.

[14] P.L. Lions and B. Mercier, Splitting Algorithms for the Sum of Two Nonlinear Operators. SIAM J. Numer. Anal. 16 (1979) 964-979.

[15] D. Quéré, Wetting and Roughness. Annu. Rev. Mater. Res. 38 (2008) 71-99.

[16] J.E. Taylor, Boundary regularity for solutions to various capillarity and free boundary problems. Commun. Partial Differ. Equ. 2 (1977) 323-357.

[17] H. Yang, A. Buguin, J.M. Taulemesse, K. Kaneko, S. Mery, A. Bergeret and P. Keller, Micron-Sized Main-Chain Liquid Crystalline Elastomer Actuators with Ultralarge Amplitude Contractions. J. Amer. Chem. Soc. 131 (2009) 15000-15004.

[18] J. Wang and B.J. Lucier, Error Bounds for Finite-Difference Methods for Rudin-Osher-Fatemi Image Smoothing. SIAM J. Numer. Anal. 49 (2011) 845-868. 